\title{
Haemoptysis: An unusual initial presentation of achalasia cardia
}

\author{
Sumit Roy Tapadar ${ }^{1}$, Jaydip Deb², Aparup Dhua ${ }^{3}$, Soumya Bhattacharya ${ }^{4}$, Tapan Das Bairagya ${ }^{5}$, Uttam Biswas ${ }^{6}$ \\ ${ }^{1}$ Assistant Professor of Department of Respiratory Medicine, R. G. Kar Medical College, Kolkata, India, ${ }^{2}$ Professor \& Head of Department of \\ Respiratory Medicine, Bankura Sammilani Medical College, Bankura, West Bengal, India, ${ }^{3}$ Assistant Professor in Department of Respiratory \\ Medicine, Midnapore Medical College, Paschim Medinipore, West Bengal, India, ${ }^{4}$ Assistant Professor of Department of Respiratory Medicine, \\ Bankura Sammilani Medical College, Bankura, West Bengal, India, ${ }^{5}$ Assistant Professor of Department of Respiratory Medicine, North Bengal \\ Medical College, Siliguri, Darjeeling, West Bengal, India, ${ }^{6}$ Clinical Tutor of Department of Medicine, College of Medicine \& JNM Hospital, \\ The West Bengal University of Health Sciences, Kalyani, Nadia, West Bengal, India
}

\section{A B S T R A C T}

A 31 year old male presented with haemoptysis, fever and cough. Chest Radiography showed patchy consolidation with breakdown in lower lobe of left lung. Further evaluation revealed the presence of achalasia cardia that resulted haemoptysis due to aspiration pneumonia. This report highlights the uncommon initial presentation of achalasia cardia in the form of haemoptysis in a previously asymptomatic individual. Although haemoptysis occurs commonly due to pulmonary disorders, it may also be an initial symptom of some rare nonpulmonary disorder. Thorough workup with strict vigilance is extremely important to elicit that type of rare aetiology.

Key words: Haemoptysis, Aspiration pneumonia, Achalasia

\section{INTRODUCTION}

Achalasia cardia usually presents with gastrointestinal symptoms like dysphagia, regurgitation and weight loss. Aspiration pneumonia may occur in patients with this abnormality. We present a rare case of achalasia cardia complicated by aspiration pneumonia with haemoptysis as the initial symptom.

\section{CASE REPORT}

A 31 year old male smoker presented with two episodes of mild haemoptysis within past two weeks along with cough and fever for the same duration. Haemoptysis was not related to food intake and the entire episode of illness was not associated with chest pain or dyspnoea. He had no co-morbidity. On examination he was febrile $\left(100^{\circ} \mathrm{F}\right)$ and had crepitations in left inter scapular region. Rest of physical examination was un-remarkable. Blood count didn't show leukocytosis $(9,600 / \mathrm{cu} \mathrm{mm})$. Chest X-ray revealed patchy consolidation with breakdown in left mid-zone.
Sputum smear examination was negative for acid fast bacilli. Computed Tomography (CT) scan of thorax showed patchy consolidation in the superior segment of left lower lobe with areas of breakdown (Figure 1). The C.T scan also revealed a fluid filled dilated oesophagus in anterior relation to vertebra and descending thoracic aorta. Another C.T section in mediastinal window, at a higher level clearly demonstrated air-fluid level. On fibre optic bronchoscopy purulent discharge was found in left lower lobe bronchus, which on pyogenic culture showed growth of Klebsiella pneumoniae. Barium swallow of oesophagus showed short segment narrowing at the distal end with proximal dilatation (Figure 2). The diagnosis of Achalasia cardia was later confirmed by oesophageal manometry, showing increased lower oesophageal sphincter pressure $(35 \mathrm{~mm} \mathrm{Hg}$.) and its non relaxation to act of swallowings. Upper G.I.endoscopy excluded any secondary cause of achalasia.

Patient was put on injection Piperacillin and Tazobactum according to the sensitivity report and he became afebrile within five days and haemoptysis was subsided within one week. Dry cough was present till second week. On follow 


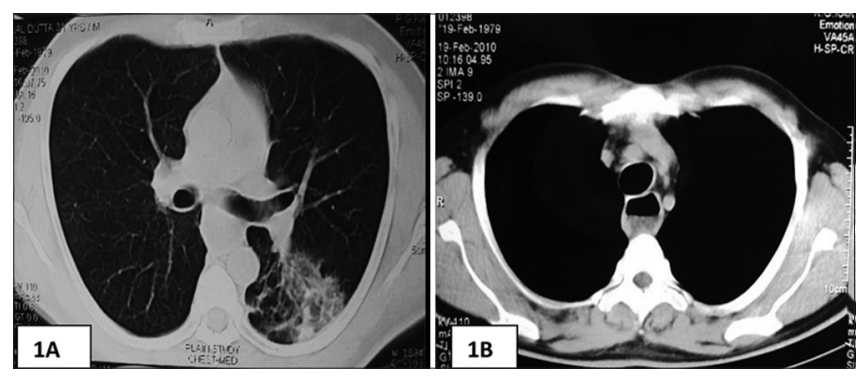

Figure 1: C.T scan of thorax: Lung window (1A) showing air space consolidation of left lower lobe with areas of breakdown and mediastinal window (1B) at higher level showing dilated oesophagus with air-fluid level

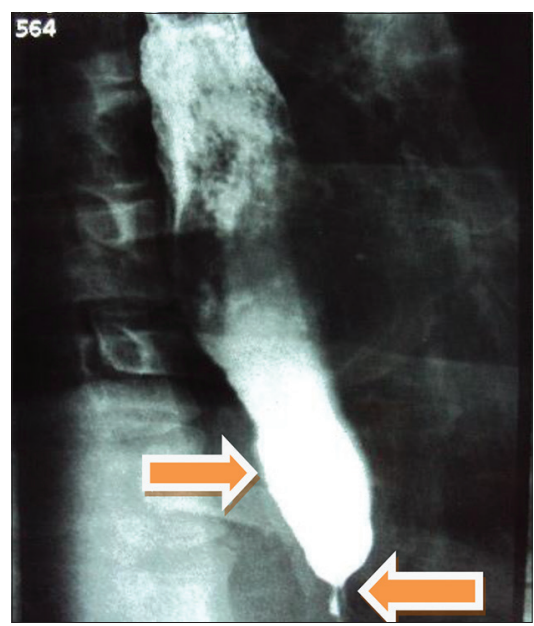

Figure 2: Barium oesophagogram showing proximal dilatation with short segment narrowing in the distal oesophagus

up, he had no fresh episode of haemoptysis and chest X ray show resolution of consolidation. Pneumatic dilatation of the affected oesophageal segment was also undertaken subsequently.

\section{DISCUSSION}

Achalasia cardia is a disease of enteric nervous system characterised by degeneration of Myenteric neurones of oesophagus, especially the lower oesophageal sphincter (LES). It is an uncommon but treatable disorder. Achalasia can manifest at any age, but commonly between 25 to 60 years, with equal gender distribution. Annual incidence of achalasia is approximately 1.6 cases per 100,000 individuals and prevalence of 10 cases per 100,000 individuals. ${ }^{1}$ In more than $90 \%$ of patients the presenting symptom is dysphagia to both solids and liquids. But a group of patients deny this symptom despite the presence of manometric and radiological features of achalasia. ${ }^{2}$ This may be due to variable reasons like loss of visceral sensation, adaptation to chronic obstruction and dilatation and absence of secondary peristalsis. The next common symptom is regurgitation (in 60\%), followed by chest pain, heart burn, weight loss and discomfort. ${ }^{3,4}$ Chest radiograph in these cases may show a homogeneous mediastinal mass/widening, air-fluid level, absence of gastric air bubble, or features of aspiration pneumonia/lung abscess, as in our case. ${ }^{4,5}$ In patients of Achalasia cardia C.T thorax commonly shows oesophageal dilatation and/or wall thickening at gastro oesophageal junction. ${ }^{6,7}$ Barium oesophagogram is an effective and time honoured tools in diagnosing achalasia and Upper G.I endoscopy helps to exclude other causes for obstruction at the lower oesophageal segment (Pseudoachalasia) but esophageal manometry is the gold standard to diagnose achalasia. ${ }^{8}$ It usually demonstrates loss of peristalsis in distal oesophagus and partial or complete loss of LES relaxation to swallows.

Contrary to usual presentation of achalasia cardia as different gastrointestinal symptom like dysphagia or regurgitation, an immunocompetent patient presented with haemoptysis from necrotizing lung lesion as a complication of aspiration due to achalasia in our case. Haemoptysis may occur in achalasia cardia due to involvement of the respiratory tree. This pulmonary involvement is typically brought about by aspiration of contaminated material from the dialated oesophagus. ${ }^{9}$ Wani et $\mathrm{al}^{10}$ reported unusual presentation of achalasia cardia as haemoptysis which was due to pulmonary actinomycosis.

\section{CONCLUSION}

Apart from different pulmonary diseases, haemoptysis may be the presenting feature of oesophageal disorder like achalasia. Similarly achalasia may have presenting signs unrelated to oesophagus. Thorough workup is necessary for specific diagnosis. CT scan of thorax may play a pivotal role not only by showing anatomical site and pattern of pulmonary involvement but also by throwing light into the other mediastinal structures to reach aetiological diagnosis.

\section{REFERENCES}

1. Sadowski DC, Ackah F, Jiang B, Svenson LW. Achalasia Incidence, Prevalence and survival. A population based study. Neurogastroenterol Motil 2010; 22;e256.

2. Blam ME, Delfyett W, Levine MS, Metz DC, Katza DA. Achalasia: A disease of varied and subtle symptoms that do not correlate with radiographic findings. Am J Gastroenterol 2002; 97: 916-923.

3. Hanbrich WS, Schaffer F, Berk JE. Bockus Gastroenterology. Philadelphia: W.B.Saunders and Co.;1995: 422-436.

4. Muralidharan S, Jairaj PS, Periyanayagam WJ, John S. Achalasia Cardia: A review of 100 cases. Aust NZ J Surg 1978; 48: 167-170.

5. Mirchandani LV, Joshi JM. Achalasia Cardia: Perplexing chest radiographs. J Assoc Physicians India 1995; 43: 721-722.

6. Tishler JM, Shin MS, Stanley RJ, Koehler RE. CT of the thorax in patients with achalasia. Dig Dis Sci 1983; 28: 692-697. 
7. Rabushka LS, Fishman EK, Kuhlman JE. CT evaluation of achalasia. J Comput Asssist Tomogr 1991; 15: 434-439.

8. Pohl D, Tutuian R. Achalasia: An overview of Diagnosis and Treatment. J Gastrointestin liv Dis 2007;10:297-303.

9. Mabeza GF, Macfarlane J. Pulmonary actinomycosis. Eur Respir
J 2003; 21: 545-551.

10. Wani AM, Hussain WM, Banjar AA, Miamini WH, Khouiah AM, Bafarai MG et al. Haemoptysis in a patient of achalasia cardia: Pulmonary actinomycosis not tuberculosis. BMJ Case Rep. 2010;2010: bcr09.2009.2287.

Authors Contribution:

SRT - Planned to report the case, Performed laboratory tests, drafted the manuscript \& reviewed the manuscript; JD - Planned to report the case \& reviewed the manuscript; AD - Planned to report the case, drafted the manuscript \& reviewed the manuscript; SB - Reviewed the manuscript; TDB - Reviewed the manuscript; UB - Reviewed the manuscript.

Source of Support: Nil, Conflict of Interest: None declared. 